\title{
Long-Term Movement Patterns and Habitat Use Of Nearshore Groundfish: Tag-Recapture in Central and Southern California Waters
}

\author{
Doyle A. Hanan* and Barbara E. Curry
}

P.O. Box 8914, Rancho Santa Fe, CA 92067 USA

\begin{abstract}
We conducted a mark-recapture tagging study evaluating long-term movement patterns and broad-scale habitat use by groundfish off southern and central California. Thirty-two species (32,366 fish) were tagged in nearshore and offshore island areas from Point Estero, California to the U.S. - Mexico border during 253 chartered fishing trips over 4 years. There were 1,569 (4.9\%) tag recoveries with recorded days at liberty (DAL) ranging 0-2,603 days (mean $288 \mathrm{~d}$ ). Observed capture and handling mortality was 1,532 fish (4.52\%). Median recovery straight-line distance was $5 \mathrm{~km}$ from original tagging site for all recaptured fish; $64 \%$ of all recaptures were greater than 100 DAL. Recovery distances of 50 $\mathrm{km}$ or more were observed for 51 fish among nine species. Longest minimum distances recorded were $510 \mathrm{~km}$ (342 DAL) by a mature olive rockfish (Sebastes serranoides) and $488 \mathrm{~km}(1,222 \mathrm{DAL})$ for an immature copper rockfish (S. caurinus). The two greatest DAL were for a brown rockfish ( $S$. auriculatus) recaptured at 2,603 DAL and $3 \mathrm{~km}$ from tag/release site and a California scorpionfish (Scorpaena guttata) recaptured at 2,126 DAL and $0.3 \mathrm{~km}$ from tag/release site. Tag recoveries, though obtained primarily in recreational fishing locations, were not spatially restricted indicating the importance of rocky reef habitat and may reflect the extent of fishable, quality habitat in southern California waters. Large sample size and tag recoveries indicate use of multiple groundfish habitats and greater movement than previously suspected.
\end{abstract}

Keywords: Channel Islands, California Bight, MPA, rockfish, mark-recapture.

\section{INTRODUCTION}

The objective of this work was to develop an understanding of long-term movement patterns and broad-scale habitat use of groundfish comprising nearshore rocky reef assemblages off central California and the southern California Bight. In collaboration with California Department of Fish and Game (CDFG) and Pacific States Marine Fisheries Commission (PSMFC) we conducted a tag and recapture program over an eight-year period using volunteer anglers aboard West Coast For Hire Sector vessels (WCFHS).

Groundfish of the family Scorpaenidae [1] including the California scorpionfish (Scorpaena guttata), and rockfish of the species-diverse genus Sebastes [2], along with several other groundfish species (e.g., lingcod, Ophiodon elongatus), which dominate nearshore and shallow shelf demersal $(<100$ $\mathrm{m})$ rocky reef fish assemblages of the California coastal ecosystem [3-5]. Groundfish species are important to recreational and commercial fisheries including the California livefish fishery [4, 6, 7]. Seven rockfish species (canary, Sebastes pinniger; darkblotched, S. crameri; widow, S. entomelas; yelloweye rockfish, $S$. ruberrimus; bocaccio, $S$. paucispinis; cowcod, S. levis; and Pacific Ocean perch, S. alutus) off the west coast of the United States are currently designated as overfished $[8,9]$.

*Address correspondence to this author at the PO Box 8914, Rancho Santa Fe, CA 92067 USA; Tel: 858-832-1159; Fax: 858-756-9268;

Emails:drhanan@cox.net,dhanan@ucsd.edu
To promote groundfish stock rebuilding and ensure fisheries yield, management measures have been implemented, as well as, additional protective measures (e.g., time and area closures, bag and size limits) [10]. Spatially based management including Essential Groundfish Habitat and Marine Protected Areas (MPA) with ecosystem based fisheries management, is being implemented and evaluated for conservation of fish and other marine populations off California and the U.S. west coast [11-14].

Implicit to the effectiveness of spatial management techniques, are the underlying habitat use and rates of movement for the protected species [15]. Mark and recapture (tagging) studies provide a means of examining broad fish movement patterns, potentially over long time periods [16]. Lowe [17] summarized the relative advantages and disadvantages of techniques used to assess fish movement and noted that tag and recapture methods are dependent upon (and may be biased by) fisheries distribution and fishing effort. In addition, capture and handling of many scorpaeniid species possessing swim bladders is complicated by physiological stress (barotrauma) that occurs as they are brought from depth to the surface [6, 18]. Methods for deflating the swim bladder, as well as, innovative in situ techniques applied to tagging of deeper water species [15, 19] are helping to lessen these problems.

Traditional tag and recapture techniques cannot provide fine-scale temporal and spatial data as acoustic tagging can provide when examining site fidelity and home range patterns [e.g. 20-22]. At this individual level, fine scale studies 


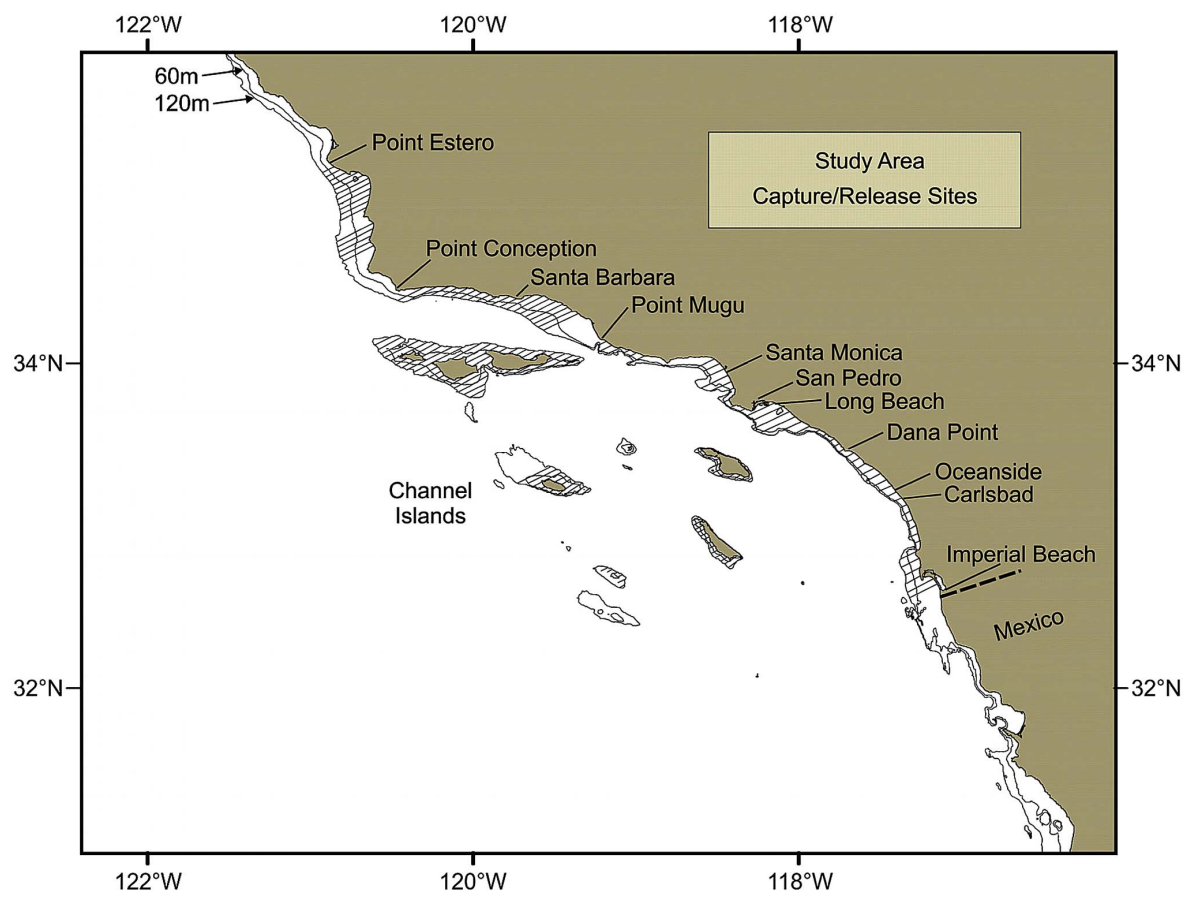

Fig. (1). Cross hatching for areas of tagging effort in central and southern California.

may represent movement of the whole population [17] but large scale tag and recapture studies may be useful for developing insight into long-term movement patterns and areas of use for the overall population or stock.

\section{MATERIALS AND METHODOLOGY}

We chartered WCFHS on which we captured and tagged groundfish along the mainland of Central California and throughout the Southern California Bight (Fig. 1). Volunteer anglers (skillful sport fishermen, chosen by WCFHS skippers) used hook and line to catch groundfish for tagging. They used a variety of fishing technics and gear configurations to catch the fish. Fishing was limited to about five anglers per fishing stop (tagging site) to control rate of fish captured and minimize fish time out of water. Anglers reeled fish to the surface slowly in an attempt to minimize barotrauma. Hyper-inflated swim bladders were deflated with 18 gauge, hypodermic needles that had been cleaned with chlorine solution [23, 24] and soaked in oxytetracycline. Each fish was measured to fork length in centimeters [25]. Fish were externally tagged using Floy Mark II tagging guns (Floy Tag \& Manufacturing, Inc., Seattle, WA) with FD-94 Floy T-bar anchor tags $(6 \mathrm{~cm}$ long). Tags were embedded in the dorsal musculature between pterygiophores with the free end of the tag slanting tail-ward. High retention rates have been demonstrated for use of the FD-94 tag among three freshwater fish species [26-28].

Data collection and tagging were conducted by scientific and technical staff.

Handling time (out of water to release) was approximately 5 minutes or less per fish and decreased during the project. Fish condition was assessed upon capture; subsequent to tagging; and upon release. Condition was recorded using a standardized, coded index based on categories of injuries, e.g. hook wound, tissue damage, bleeding, and fin damage [29]. Condition was broadly categorized as good no eye damage, stomach and anus intact; moderate - evidence of eye distention but no crystallization; slight stomach distention; or poor - both eyes damaged with evidence of crystallization, bleeding, stomach or anus distention. Fish in poor condition were placed in holding tanks for up to 15 minutes for observation, recovery, and release. If the fish was not suitable for release (decided by tagging technician) they were sacrificed; if tagged and tag salvageable, tags were reused.

Observed mortality (determined by tagging technician as a fish that appeared lifeless and unresponsive to touch) was assessed and occurrence pre-tagging or post-tagging was recorded. Post-tagging mortality does not account for any mortality that occurred after fish were released and unobservable by technician or crew.

An informational flyer offing a reward for tagged fish was periodically distributed at landing sites and docking facilities to alert recreational and commercial fishermen regarding the study. Tags were individually numbered and marked "CDFG REWARD 562-342-7100." Reward was a letter describing tagging location, date, and fish length when tagged, along with a "Nearshore Groundfish Tagging Project" t-shirt displaying fish illustrations and logos of the participating agencies on the back.

For these analyses, we used recovery information received from November 2002 to August 2010 but recovery data are continuing to be received (i.e., 5 March 2011, vermilion rockfish (S. miniatus) recaptured at $111 \mathrm{~km} ; 2,104$ DAL). Recoveries were reported to D. A. Hanan and E. W. Roberts or other CDFG personnel which allowed verification with reporting fishermen; tags were also returned by mail.

We estimated total observed mortality by species as the sum of all fish that died (pre- and post- tagging mortality). Tag recovery rates were computed as number recovered di- 


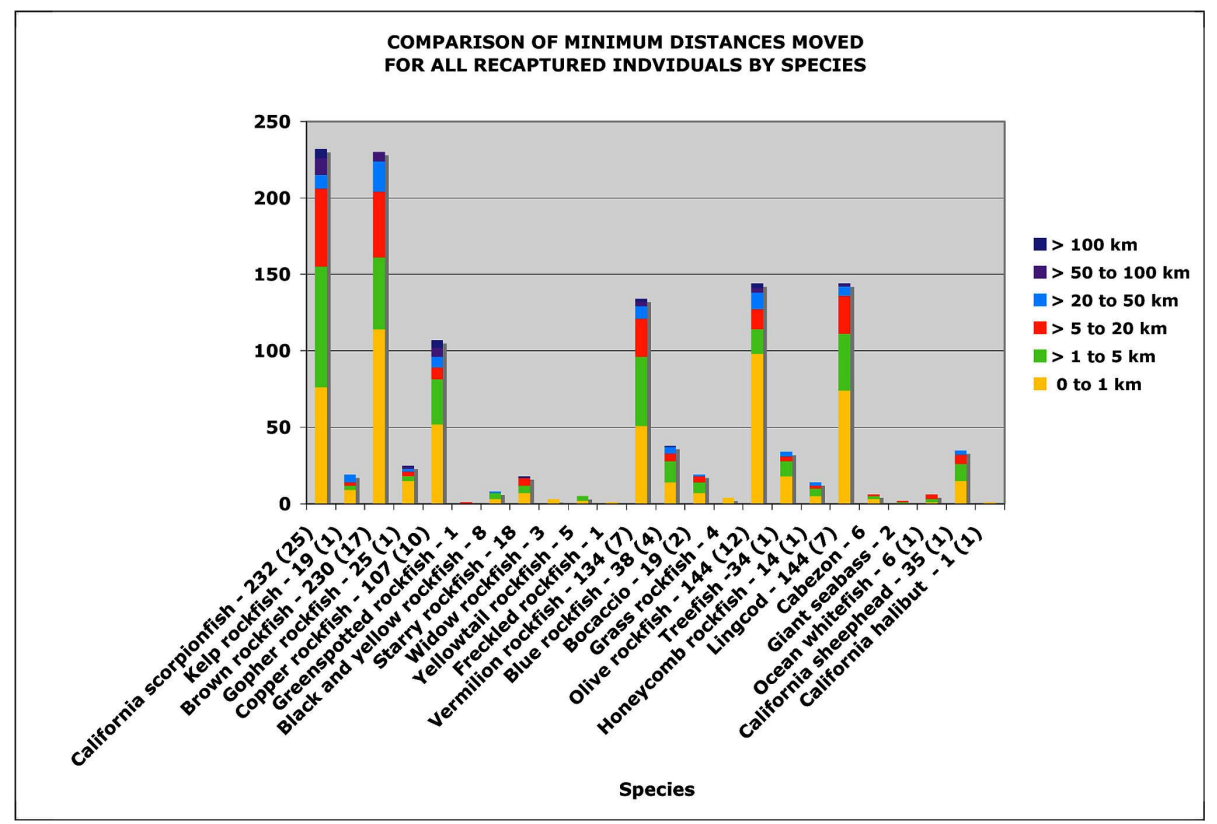

Fig. (2). Minimum distance moved with increments according to legend on right. Total recoveries follow common name; those with no distance estimate in parentheses.

vided by number tagged. Average length of tagged fish was calculated by species. Approximate maturity was estimated based on published results of maturity ogives and, when possible, using ogives specific to California waters. Straight-line recapture distance was calculated to nearest nautical mile and then converted to kilometers. We summarized distances within increments of $<=1 \mathrm{~km},>1$ to $<=5 \mathrm{~km},>5$ to $<=20 \mathrm{~km}$, $>20$ to $<=50 \mathrm{~km},>50$ to $<=100 \mathrm{~km}$, and $>100 \mathrm{~km}$ (Fig. 2).

DAL and distance from tagging site were plotted and linear regression of distance on DAL were computed for species with recapture distances $>50 \mathrm{~km}$ and sample size 25 recoveries or greater. We accept that displacement resulting from capture and release may have affected movement of some tagged fish.

\section{RESULTS}

Scorpionfish, rockfish of the family Scorpaenidae, and six other fish species were tagged during the tagging study period, 21 November 2002 to 24 July 2006. A total of 253 trips with the WCFHS were chartered for tagging in nearshore areas (including seven State Recreational Groundfish Management Areas [10], several State Marine Reserves along the mainland, and the Channel Islands Marine Protected Areas [30]) from Point Estero, California to waters off Imperial Beach, California near the U.S. - Mexico border (Fig. 1). Among fishing locations, there were a total of 1,873 tagging sites (vessel stops) with corresponding latitude and longitude coordinates and water depths ranging from 4 to $116 \mathrm{~m}$ (mean $29 \mathrm{~m}$ ). We coordinated with WCFHS skippers to choose locations where groundfish were likely to be caught and in areas with water depth tended to be less than $100 \mathrm{ft}(30.5 \mathrm{~m})$.

A total of 33,882 fish were caught, representing 32 species (scorpionfish, 25 rockfish species, and nearshore species: lingcod, cabezon (Scorpaenichthys marmoratus), giant seabass (Stereolepis gigas), ocean whitefish (Caulolatilus princeps), California sheephead (Semicossyphus pulcher),
California halibut (Paralichthys californicus), (Table 1). A total of 32,366 fish were tagged and released. There were $1,569(4.9 \%)$ reports of tagged fish recaptured or observed. Of these, 1,321 reports had recapture information sufficient for analysis. Recapture and then re-release on the same day as tagged was considered zero (0) DAL. On the day of tagging, 193 fish were recaptured (0 DAL) and re-released. These 193 fish were of 11 species, predominately California scorpionfish ( $n=95)$ and lingcod $(n=69)$. Twelve of the 193 fish were recaptured a third time at a later date (23-607 DAL). The two greatest DAL were for a brown rockfish ( $S$. auriculatus; $2,603 \mathrm{DAL}$ ) recaptured $3 \mathrm{~km}$ from tag/release site and a California scorpionfish $(2,126 \mathrm{DAL})$ recaptured $0.3 \mathrm{~km}$ from tag/release site.

The majority of tag recoveries were made by recreational fishermen (private vessels and WCFHS) but a few recoveries $(<100)$ were from commercial vessels, kayakers, and divers (including underwater visual tag observations and video by divers). Recovery location was based on exact coordinates if available but occasionally by CDFG fisheries statistical catch areas (Fish and Game reporting blocks). Some tagged fish were recaptured; then re-released with the tag intact and recovery information reported by the fishermen. Data on disposition of fish subsequent to first recapture and rerelease are inconsistent; therefore, the total number of tagged fish recaptured and retained and total number of tagged fish re-released are not known with certainty. A total of 53 fish were confirmed to have been recaptured at least two times after day of tagging, and four of these were recaptured a third time (Table 2). One treefish (S. serriceps) was recaptured four times (the four DAL periods in chronological order were: $70,16,175$, and 537 ; total DAL to last capture $=$ 798).

Sex of most fish tagged was not known with certainty; although, average fork length of tagged fish (Table 1) exceeded length at sexual maturity for California scorpionfish, greenspotted rockfish, black and yellow rockfish, bocaccio, 
Table 1. Summary of All Species Tagged in Nearshore Central and Southern California Waters and Offshore Island Areas Between November 2002 and July 2006, and Recovery Data Through August 2010

\begin{tabular}{|c|c|c|c|c|c|c|c|}
\hline Species & $\begin{array}{c}\text { Number } \\
\text { Tagged }\end{array}$ & $\begin{array}{c}\text { Number } \\
\text { Recoveries }\end{array}$ & $\begin{array}{c}\text { Percent } \\
\text { Recovered }\end{array}$ & $\begin{array}{c}\text { Fork Length at } \\
\text { Tagging } \\
\text { (Average } \mathrm{cm} \text { ) }\end{array}$ & $\begin{array}{c}\text { Fork } \\
\text { Length } \\
(\text { Range cm) }\end{array}$ & $\begin{array}{c}\text { Average Days } \\
\text { at Liberty } \\
\text { (SD) }\end{array}$ & $\begin{array}{l}\text { Days at } \\
\text { Liberty } \\
\text { (Range) }\end{array}$ \\
\hline $\begin{array}{c}\text { California scor- } \\
\text { pionfish }\end{array}$ & 2751 & 257 & $9.3 \%$ & $27.5(n=2743)$ & $12.9-43.5$ & $408.8(431.6)$ & $2-2126$ \\
\hline Kelp rockfish & 1746 & 20 & $1.2 \%$ & $30.0(n=1731)$ & $18.0-45.6$ & $157.5(142.7)$ & $14-415$ \\
\hline Brown rockfish & 1453 & 247 & $17.0 \%$ & $27.3(n=1448)$ & $13.7-47.5$ & $204.7(278.0)$ & $1-2603$ \\
\hline Gopher rockfish & 2709 & 26 & $1.0 \%$ & $25.7(n=2685)$ & $11.6-51.3$ & $352.6(388.9)$ & $14-1650$ \\
\hline Copper rockfish & 2828 & 117 & $4.1 \%$ & $32.2(n=2821)$ & $13.4-61.0$ & 441.5 (396.6) & $11-2052$ \\
\hline $\begin{array}{l}\text { Greenspotted } \\
\text { rockfish }\end{array}$ & 56 & 1 & $1.8 \%$ & $28.3(n=55)$ & $20.4-34.2$ & 560 & 560 \\
\hline $\begin{array}{c}\text { Black and yellow } \\
\text { rockfish }\end{array}$ & 115 & 8 & $7.0 \%$ & $25.5(n=114)$ & $11.0-34.2$ & $322.9(311.3)$ & $38-824$ \\
\hline Starry rockfish & 478 & 18 & $3.8 \%$ & $28.1(n=470)$ & $13.5-49.4$ & $457.0(315.2)$ & $30-1263$ \\
\hline Calico rockfish & 82 & 0 & $0.0 \%$ & $14.4(82)$ & $10.9-21.0$ & - & - \\
\hline Widow rockfish & 128 & 3 & $2.3 \%$ & $29.7(n=128)$ & $11.4-43.4$ & 203 & 203 \\
\hline $\begin{array}{l}\text { Yellowtail rock- } \\
\text { fish }\end{array}$ & 71 & 5 & $7.0 \%$ & $26.2(\mathrm{n}=71)$ & $13.3-35.0$ & $233.2(79.7)$ & $159-354$ \\
\hline $\begin{array}{l}\text { Squarespot rock- } \\
\text { fish }\end{array}$ & 76 & 0 & $0.0 \%$ & $20.0(n=76)$ & $13.7-30.2$ & - & - \\
\hline Freckled rockfish & 6 & 1 & $17.0 \%$ & $22.7(n=6)$ & $21.2-25.5$ & 52 & 52 \\
\hline Black Rockfish & 15 & 0 & $0.0 \%$ & $28.3(n=15)$ & $20.5-26.4$ & - & - \\
\hline $\begin{array}{l}\text { Vermilion rock- } \\
\text { fish }\end{array}$ & 3161 & 141 & $4.5 \%$ & $34.5(n=3149)$ & $12.6-85.1$ & $455.5(415.86)$ & $3-1963$ \\
\hline Blue rockfish & 5221 & 42 & $0.8 \%$ & $28.1(n=5189)$ & $11.3-63.2$ & $339.7(295.0)$ & $42-1460$ \\
\hline China rockfish & 7 & 0 & $0.0 \%$ & $29.1(n=7)$ & $25.1-34.0$ & - & - \\
\hline Bocaccio & 454 & 21 & $4.6 \%$ & $43.7(n=449)$ & $11.5-63.0$ & $529.5(424.1)$ & $51-1558$ \\
\hline Canary rockfish & 13 & 0 & $0.0 \%$ & $30.0(n=13)$ & $25.7-34.1$ & - & - \\
\hline Grass rockfish & 26 & 4 & $15.4 \%$ & $31.4(n=26)$ & $22.0-43.9$ & $\begin{array}{c}34.8 \\
(43.8)\end{array}$ & $16-99$ \\
\hline Rosy rockfish & 131 & 0 & $0.0 \%$ & $19.9(n=29)$ & $14.4-29.6$ & - & - \\
\hline Flag rockfish & 22 & 0 & $0.0 \%$ & $25.9(n=21)$ & $16.2-34.1$ & - & - \\
\hline Olive rockfish & 3278 & 156 & $4.8 \%$ & $29.9(n=3255)$ & $12.4-57.8$ & $129.6(128.9)$ & $1-866$ \\
\hline Treefish & 945 & 35 & $3.7 \%$ & $28.2(n=940)$ & $13.5-39.9$ & $436.9(395.8)$ & $4-1780$ \\
\hline $\begin{array}{l}\text { Honeycomb rock- } \\
\text { fish }\end{array}$ & 610 & 15 & $2.5 \%$ & $19.4(n=607)$ & $11.6-30.2$ & $540.8(462.1)$ & $67-1849$ \\
\hline Kelp greenling & 38 & 0 & $0.0 \%$ & $32.8(n=37)$ & $25.5-46.6$ & - & - \\
\hline Lingcod & 3865 & 151 & $3.9 \%$ & $56.0(n=3853)$ & $21.3-94.5$ & $287.3(323.6)$ & $1-1791$ \\
\hline Cabezon & 300 & 6 & $2.0 \%$ & $39.2(n=300)$ & $18.4-59.4$ & $112(108.2)$ & $3-304$ \\
\hline Giant seabass & 14 & 2 & $14.3 \%$ & $65.4(n=14)$ & $41.7-102.2$ & 742.5 (703.6) & $245-1240$ \\
\hline Ocean whitefish & 210 & 7 & $3.4 \%$ & $38.6(n=208)$ & $25.7-65.0$ & $536.3(699.0)$ & $84-2085$ \\
\hline $\begin{array}{l}\text { California } \\
\text { sheephead }\end{array}$ & 1485 & 36 & $2.4 \%$ & $38.2(n=1476)$ & $19.5-75.6$ & $402.5(440.3)$ & $7-1721$ \\
\hline California halibut & 72 & 2 & $2.8 \%$ & $60.2(n=68)$ & $31.1-113.5$ & $194.5(75.7)$ & $141-248$ \\
\hline
\end{tabular}


Table 2. Fish Tagged, Recaptured, and Re-Released by Fishermen with Tag in Place

\begin{tabular}{|c|c|c|c|c|c|c|}
\hline Species & $\begin{array}{l}\text { Number Fish } \\
\text { Second } \\
\text { Recapture }\end{array}$ & $\begin{array}{l}\text { Days at Liberty } \\
\text { (Range } \\
\text { Capture } 1 \text { and 2) }\end{array}$ & $\begin{array}{c}\text { Number Fish } \\
\text { Third Recapture }\end{array}$ & $\begin{array}{c}\begin{array}{c}\text { Days at Liberty } \\
\text { (Range } \\
\text { Capture3) }\end{array} \\
\end{array}$ & $\begin{array}{c}\text { Number Fish } \\
\text { Fourth Recapture }\end{array}$ & $\begin{array}{l}\text { Days at Liberty } \\
\text { (Range Capture 4) }\end{array}$ \\
\hline California scorpionfish & 10 & $11-1486$ & 0 & - & 0 & - \\
\hline Brown rockfish & 18 & $8-1228$ & 2 & $67-95$ & 0 & - \\
\hline Gopher rockfish & 2 & $24-196$ & 0 & - & 0 & - \\
\hline Vermilion rockfish & 4 & $95-528$ & 1 & 118 & 0 & - \\
\hline Blue rockfish & 2 & $504-909$ & 0 & - & 0 & - \\
\hline Olive rockfish & 8 & $15-228$ & 3 & $104-138$ & 0 & - \\
\hline Treefish & 2 & $16-196$ & 1 & 261 & 1 & 537 \\
\hline Lingcod & 5 & $48-389$ & 0 & - & 0 & - \\
\hline California sheephead & 2 & $99-487$ & 0 & - & 0 & - \\
\hline
\end{tabular}

Table 3. Observed Mortality for Fish by Species Caught in this Tagging Study

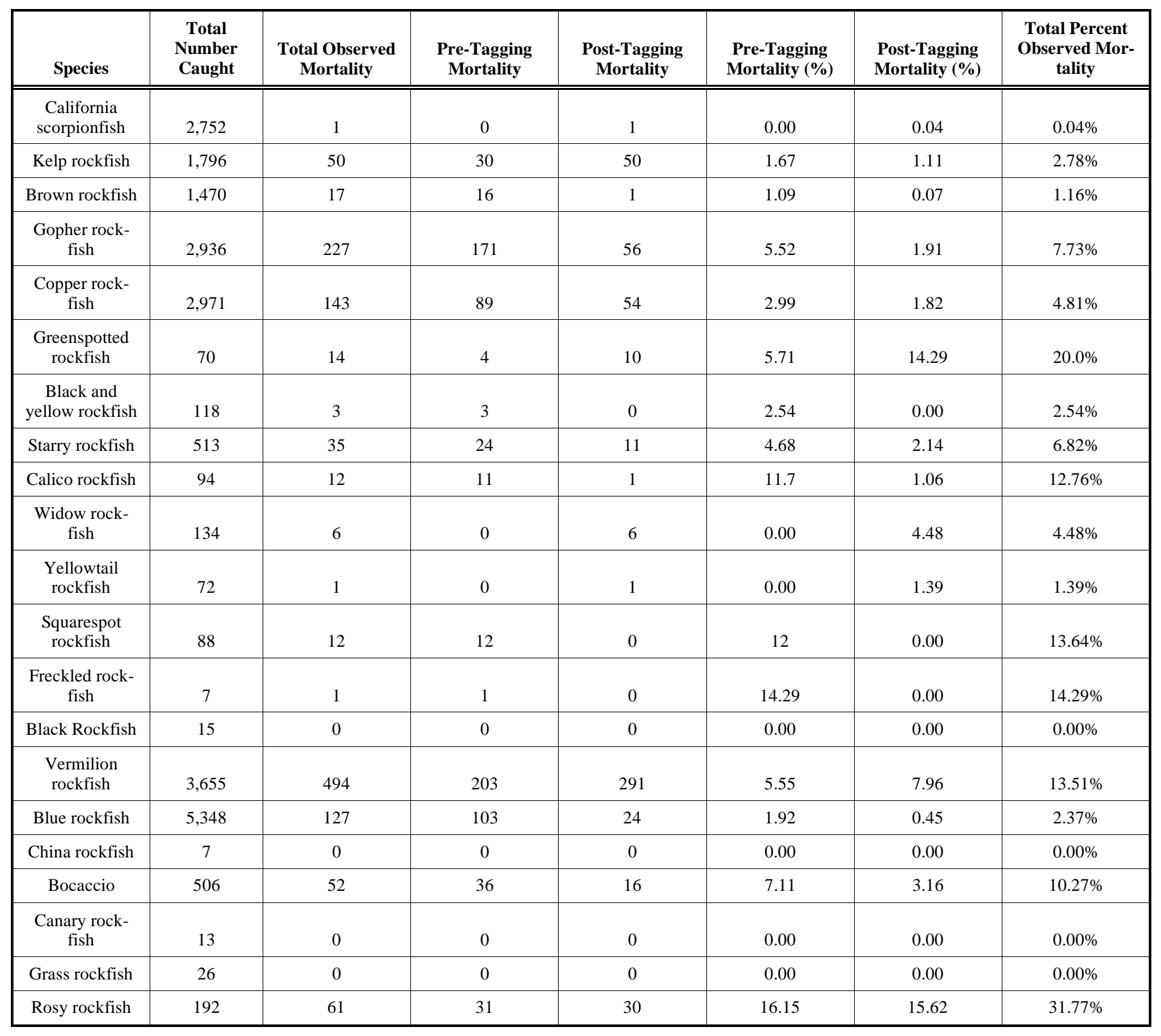


Table 3. cont....

\begin{tabular}{|c|c|c|c|c|c|c|c|}
\hline Species & $\begin{array}{c}\text { Total } \\
\text { Number } \\
\text { Caught }\end{array}$ & $\begin{array}{l}\text { Total Observed } \\
\text { Mortality }\end{array}$ & $\begin{array}{c}\text { Pre-Tagging } \\
\text { Mortality }\end{array}$ & $\begin{array}{c}\text { Post-Tagging } \\
\text { Mortality }\end{array}$ & $\begin{array}{c}\text { Pre-Tagging } \\
\text { Mortality (\%) }\end{array}$ & $\begin{array}{l}\text { Post-Tagging } \\
\text { Mortality (\%) }\end{array}$ & $\begin{array}{c}\text { Total Percent } \\
\text { Observed Mor- } \\
\text { tality }\end{array}$ \\
\hline Flag rockfish & 26 & 4 & 3 & 1 & 11.54 & 3.84 & $15.38 \%$ \\
\hline Olive rockfish & 3,412 & 134 & 89 & 45 & 2.61 & 1.32 & $3.93 \%$ \\
\hline Treefish & 981 & 36 & 28 & 8 & 2.85 & 0.82 & $3.67 \%$ \\
\hline $\begin{array}{l}\text { Honeycomb } \\
\text { rockfish }\end{array}$ & 689 & 79 & 38 & 41 & 4.68 & 2.14 & $11.47 \%$ \\
\hline Kelp greenling & 39 & 1 & 0 & 1 & 0.00 & 2.56 & $2.56 \%$ \\
\hline Lingcod & 3,871 & 6 & 6 & 0 & 6.00 & 0.00 & $0.15 \%$ \\
\hline Cabezon & 300 & 0 & 0 & 0 & 0.00 & 0.00 & $0.00 \%$ \\
\hline Giant seabass & 14 & 0 & 0 & 0 & 0.00 & 0.00 & $0.00 \%$ \\
\hline $\begin{array}{l}\text { Ocean white- } \\
\text { fish }\end{array}$ & 210 & 0 & 0 & 0 & 0.00 & 0.00 & $0.00 \%$ \\
\hline $\begin{array}{l}\text { California } \\
\text { sheephead }\end{array}$ & 1,485 & 16 & 8 & 8 & 0.54 & 0.54 & $1.08 \%$ \\
\hline $\begin{array}{c}\text { California } \\
\text { halibut }\end{array}$ & 72 & 0 & 0 & 0 & 0.00 & 0.00 & $0.00 \%$ \\
\hline $\begin{array}{l}\text { OVERALL } \\
\text { TOTAL }\end{array}$ & 33,882 & 1,532 & 906 & 656 & 2.7 & 1.9 & $4.5 \%$ \\
\hline
\end{tabular}

Table 4. Details of Nine Fish Species with Estimated Distances from Tagging/Release Site to Recapture Site of $50 \mathrm{~km}$ or Greater. Abbreviations: A-Neither Eye Distended, C-Both Eyes Distended, D-One Eye Crystallized, E-Both Eyes Crystallized, F-Stomach Extended into Mouth, H-Hook Wound Minor, I-Hook Wound Severe, L-Bleeding, R-Active, X-Abdomen Swollen, West Coast For Hire Sector (WCFHS)

\begin{tabular}{|c|c|c|c|c|c|c|c|c|c|}
\hline Species & Tag & $\begin{array}{c}\text { Capture } \\
\text { Habitat } \\
\text { and Depth } \\
\text { (m) }\end{array}$ & $\begin{array}{l}\text { Length at } \\
\text { Tagging } \\
\text { (cm) }\end{array}$ & $\begin{array}{l}\text { Estimated } \\
\text { Maturity }\end{array}$ & $\begin{array}{c}\text { Fish } \\
\text { Condition at } \\
\text { Release }\end{array}$ & $\begin{array}{l}\text { Days at } \\
\text { Liberty }\end{array}$ & $\begin{array}{c}\text { Distance } \\
\text { Moved (km) }\end{array}$ & $\begin{array}{c}\text { Rate of } \\
\text { Movement } \\
\text { (km/Day) }\end{array}$ & $\begin{array}{c}\text { Re-Capture } \\
\text { Vessel }\end{array}$ \\
\hline \multirow[t]{12}{*}{$\begin{array}{l}\text { California } \\
\text { scorpionfish }\end{array}$} & 01820 & rocky; 15 & 30.1 & M & good & 224 & 197 & 0.88 & $\begin{array}{l}\text { Commercial } \\
\text { trawl }\end{array}$ \\
\hline & 02533 & rocky; 16 & 30.4 & M & good & 92 & 200 & 2.17 & $\begin{array}{c}\text { Commercial } \\
\text { trawl }\end{array}$ \\
\hline & 02546 & rocky; 16 & 25.8 & M & good & 197 & 200 & 1.02 & $\begin{array}{c}\text { Commercial } \\
\text { trawl }\end{array}$ \\
\hline & 04619 & $\begin{array}{l}\text { artificial } \\
\text { reef; } 22\end{array}$ & 19.6 & M & good, A, R & 1788 & 53 & 0.03 & $\begin{array}{l}\text { Recreational } \\
\text { - WCFHS }\end{array}$ \\
\hline & 05173 & $\begin{array}{c}\text { artificial } \\
\text { reef; } 22\end{array}$ & 23.5 & M & good, A & 515 & 94 & 0.18 & $\begin{array}{l}\text { Recreational } \\
\text { - WCFHS }\end{array}$ \\
\hline & 11561 & $\begin{array}{c}\text { rocky, kelp; } \\
26\end{array}$ & 31.5 & M & good, A & 810 & 171 & 0.21 & $\begin{array}{l}\text { Recreational } \\
\text { - WCFHS }\end{array}$ \\
\hline & 11981 & rocky; 26 & 24.4 & M & good & 187 & 53 & 0.28 & $\begin{array}{c}\text { Commercial } \\
\text { trawl }\end{array}$ \\
\hline & 16571 & rocky; 29 & 30.4 & M & good & 511 & 173 & & $\begin{array}{l}\text { Recreational } \\
\text { - WCFHS }\end{array}$ \\
\hline & 17155 & $\mathrm{NA} ; 29$ & 29.1 & M & good & 442 & 80 & 0.18 & $\begin{array}{l}\text { Recreational } \\
\text { - WCFHS }\end{array}$ \\
\hline & 17199 & NA; 29 & 26.0 & M & good & 555 & 70 & 0.13 & $\begin{array}{l}\text { Recreational } \\
\text { - WCFHS }\end{array}$ \\
\hline & 17204 & NA; 29 & 29.0 & M & good & 68 & 70 & 1.03 & $\begin{array}{l}\text { Recreational } \\
\text { - WCFHS }\end{array}$ \\
\hline & 27543 & $\begin{array}{l}\text { artificial } \\
\text { reef; } 43\end{array}$ & 28.0 & M & good, H, R & 1022 & 68 & 0.07 & Not known \\
\hline
\end{tabular}


Table 4. cont....

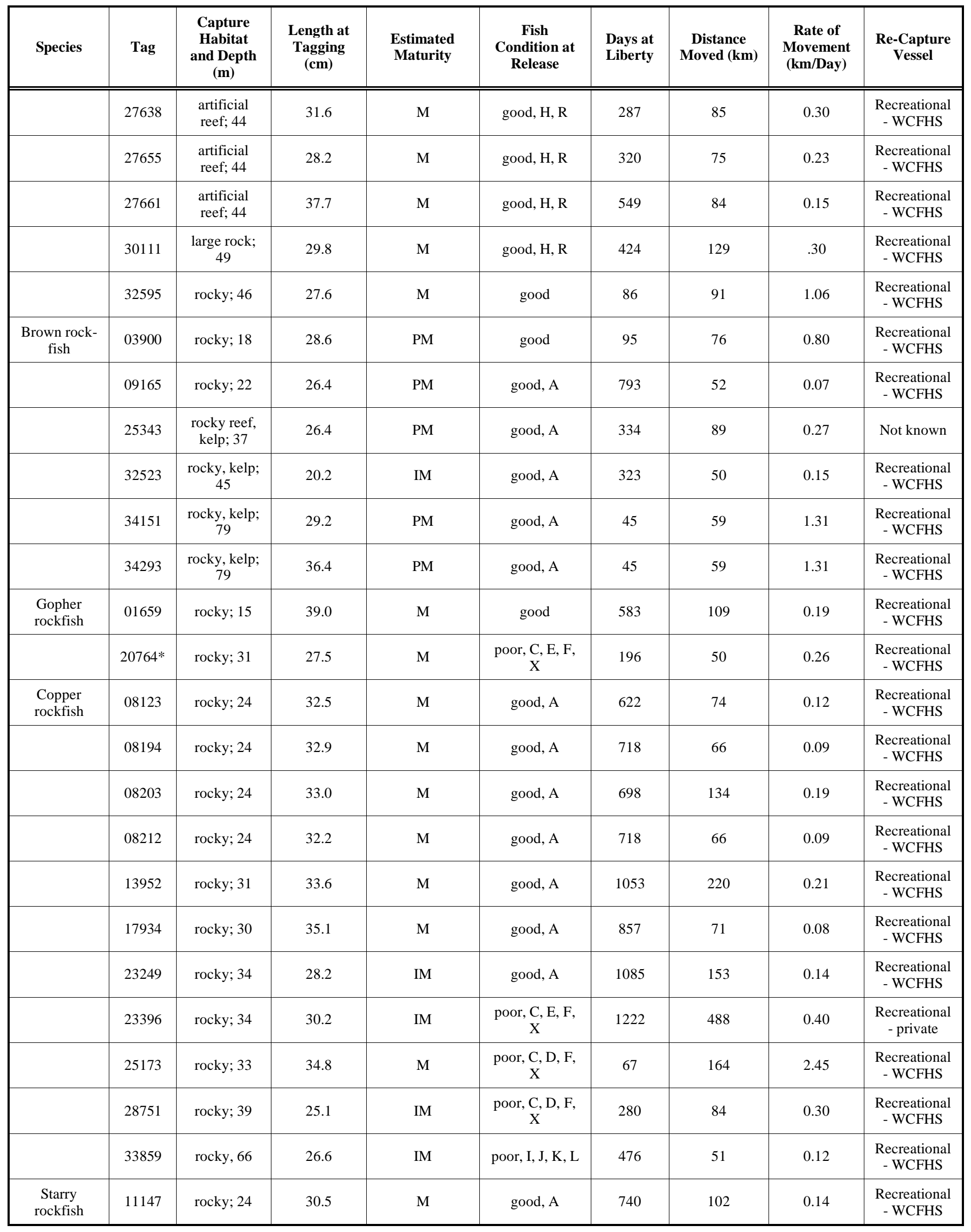


Table 4. cont.....

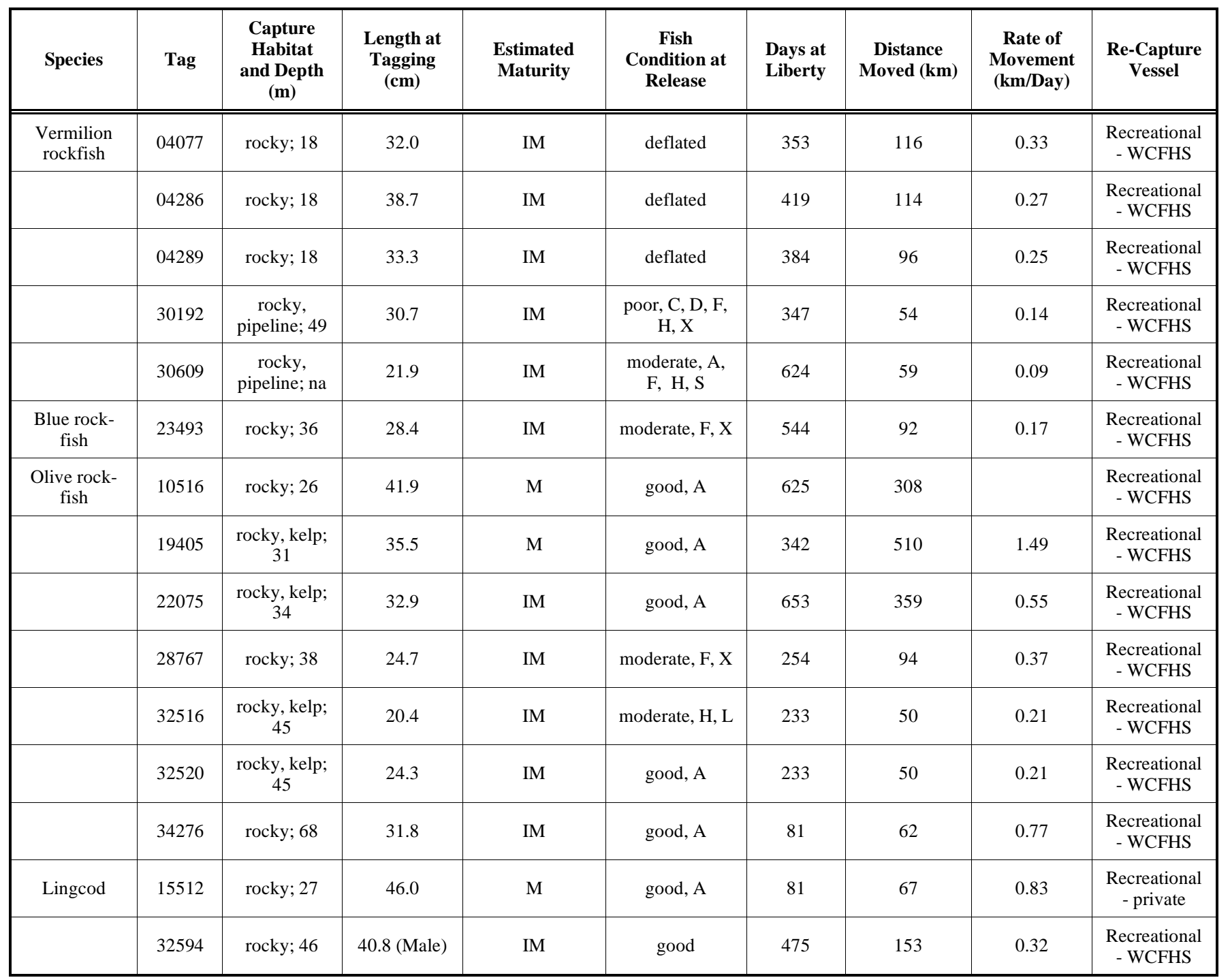

grass rockfish, honeycomb rockfish, and kelp greenling (Hexagrammos decagrammus) [4, 31-35].

Total observed mortality was 1,532 fish, $4.5 \%$ of fish caught. Pre-tagging mortality was higher at 906 fish $(2.7 \%)$ than post-tagging mortality 656 fish (1.9\%). Observed mortality was highest for rosy rockfish (Sebastes rosaceus; $31.8 \%)$, high for green- spotted rockfish (S. chlorostictus; $20 \%$ ), and lowest for California scorpionfish (0.04\%). Mortality was not observed for: black ( $S$. melanops), china $(S$. nebulosus), nor canary rockfish; not observed for lingcod, cabezon, giant seabass, ocean whitefish, nor California halibut. Pre-tagging mortality was relatively high for rosy rockfish $(16.5 \%)$ and freckled rockfish (S. lentiginosus, $14.3 \%)$. Post-tagging mortality was relatively high for rosy $(15.6 \%)$ and greenspotted (14.3\%) rockfish (Table 3 ).

For all recaptured fish about $50 \%$ were recovered at distances greater than $5 \mathrm{~km}$ from the original tag/release sites; the number of fishes recaptured diminished with increasing distance from tagging/release site (Fig. 2). Long distance movements of $50 \mathrm{~km}$ or more were observed among 9 spe- cies (Table 4). The longest straight line distances estimated were $488 \mathrm{~km}$ for copper (Sebastes caurinus) and $510 \mathrm{~km}$ for a mature olive rockfish ( $S$. serranoides).

Linear regression analysis revealed no significant correlation between DAL and distance from tagging site for 8 species tested (brown, gopher; copper, vermilion, blue, olive rockfish, California scorpionfish, and lingcod).

Individuals from 9 species moved $50 \mathrm{~km}$ or more. Fish of 7 species (California scorpionfish, $\mathrm{n}=4$; gopher rockfish, $\mathrm{n}=1$; copper rockfish, $\mathrm{n}=4$; starry rockfish, $\mathrm{n}=1$; vermilion rockfish, $n=4$, blue, $n=1$, and olive rockfish, $n=4$ ) tagged in nearshore areas were recaptured offshore or at offshore islands as described below.

Of 257 California scorpionfish recaptures, $155(67 \%)$ were within $5 \mathrm{~km}$ of their original tag/release site $(2-2,126$ DAL) and $76(33 \%)$ of these were within $1 \mathrm{~km}$ of the tagging site. California scorpionfish were caught primarily in areas nearshore between Pt. Mugu and San Diego, and near the Channel Islands, which includes possible important Southern California spawning grounds (Santa Monica Bay, Dago 


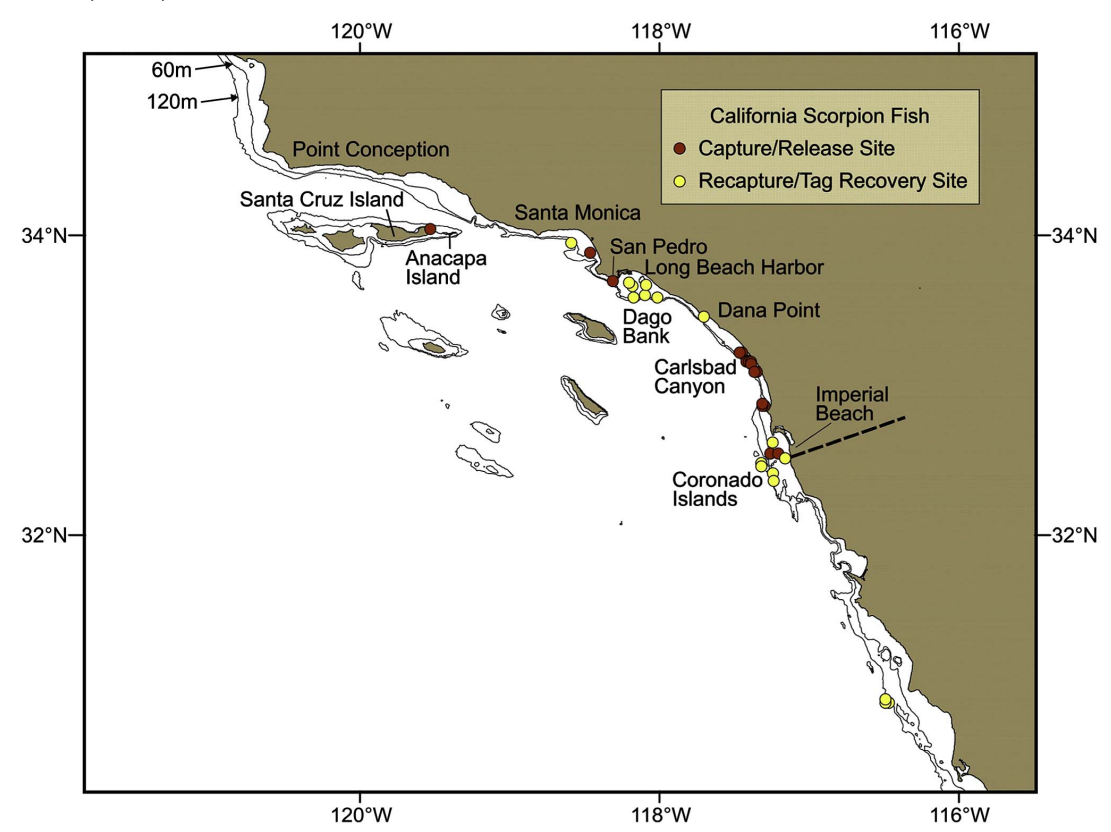

Fig. (3). Tag and recovery sites for 17 tagged mature California scorpionfish (Sebastes guttata) with movement of at least $50 \mathrm{~km}$ off southern Calif. and Baja Calif., Mexico.

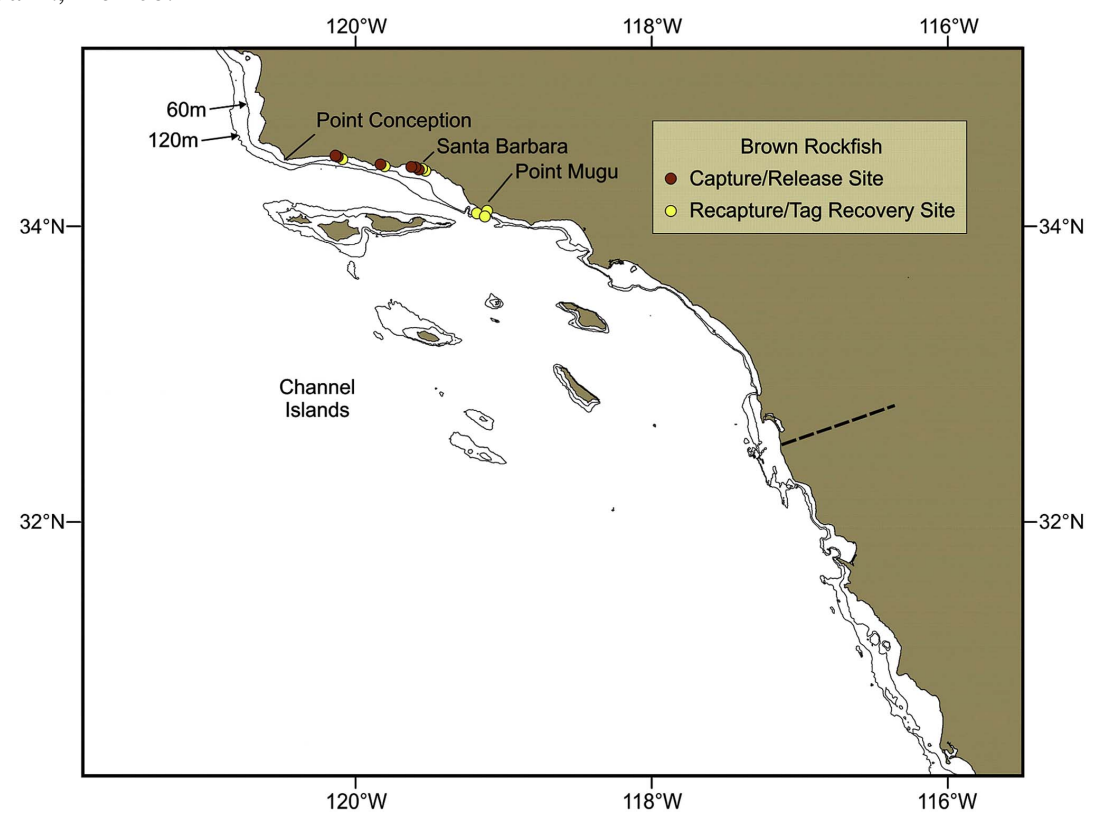

Fig. (4). Tag and recovery sites for 6 brown rockfish (Sebastes auriculatus) with movements of at least $50 \mathrm{~km}$.

Bank near Long Beach Harbor, Anacapa Island, and the Coronado Islands [31]). Seventeen mature [31] California scorpionfish were recaptured at distances of $50 \mathrm{~km}$ or more (68 - 1,788 DAL) and 7 of these fish were recaptured at Dago Bank off Long Beach Harbor during May to December. Movements of fish among spawning grounds were observed. A scorpionfish tagged in April between Santa Cruz and Anacapa Islands was recaptured the following June off Long Beach (129 km, 424 DAL). Seven scorpionfish had recapture distances up to $200 \mathrm{~km}$ along coastal areas between Carlsbad and Baja California. Recoveries include 1 fish tagged in February off San Pedro, approximately $16 \mathrm{~km}$ from Dago Bank, which was recaptured off the Coronado Islands, Baja California (just south of the U.S.-Mexico border) in July (511 DAL; Fig. 3, Table 4). The most rapid movement rates from tagging/release sites to recapture sites were ob- served for 3 mature California scorpionfish averaging speeds of 1.0, 1.1, and $2.2 \mathrm{~km} /$ day (Table 4).

The majority $(n=161 ; 70 \%)$ of recaptured brown rockfish were within $5 \mathrm{~km}$ of the tag/release site and $50 \%$ were within $1 \mathrm{~km}(2$ - 2,603 DAL). Long distance movements $(>50 \mathrm{~km})$ were observed for 6 brown rockfish and included movements along shore of up to $89 \mathrm{~km}$ (45 - 793 DAL) between points north of Santa Barbara to off Pt. Mugu (Fig. 4, Table 4). Movement rates $>1 \mathrm{~km} /$ day were observed for two brown rockfish.

Fifteen $(60 \%)$ of the recaptured gopher rockfish $(S$. carnatus) were within $1 \mathrm{~km}$ of the tag/release sites (19 - 636 DAL). One mature [33] gopher rockfish moved $109 \mathrm{~km}$ from east of Santa Rosa Island to nearshore south of Pismo Beach in Central California (583 DAL, Fig. 5, Table 4). 


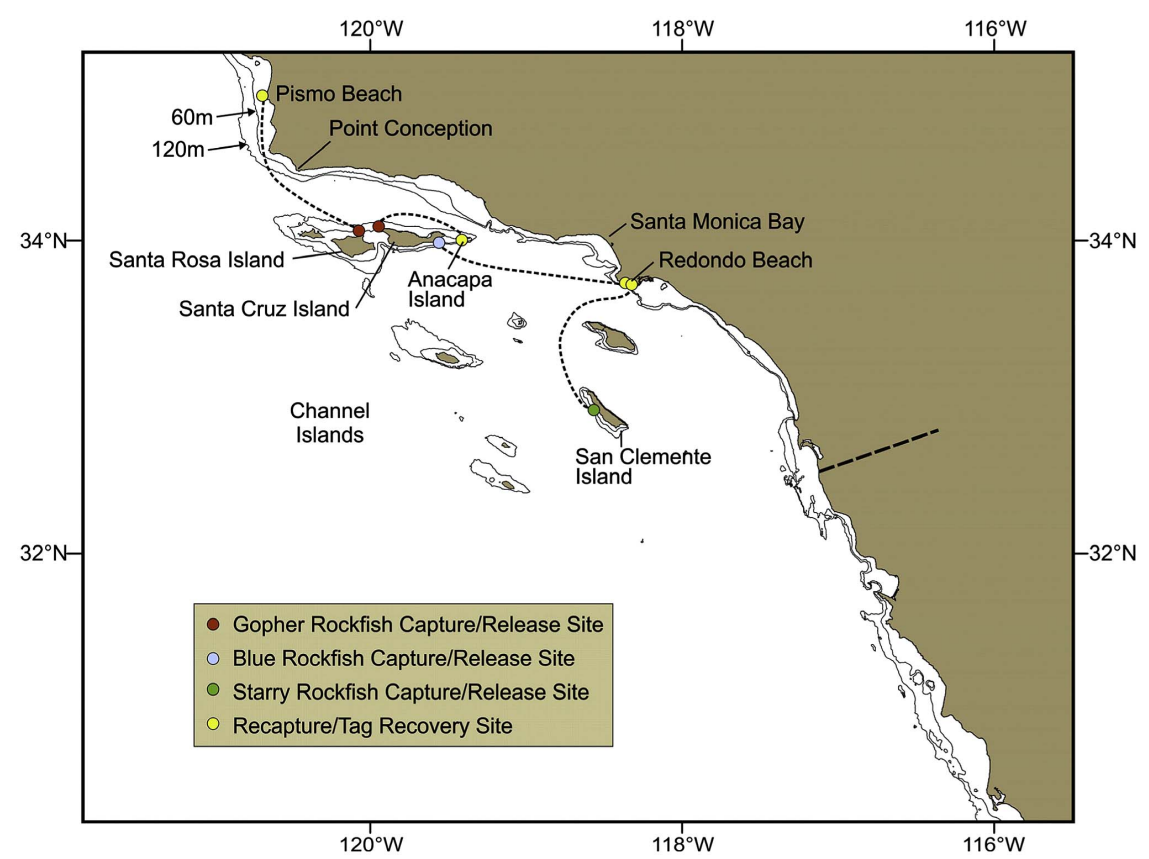

Fig. (5). Tag and recovery sites of a mature gopher (Sebastes carnatus; $109 \mathrm{~km})$, a mature starry (S. constellatus; $102 \mathrm{~km})$, and a mature blue rockfish (S. mystinus; $92 \mathrm{~km}$ ).

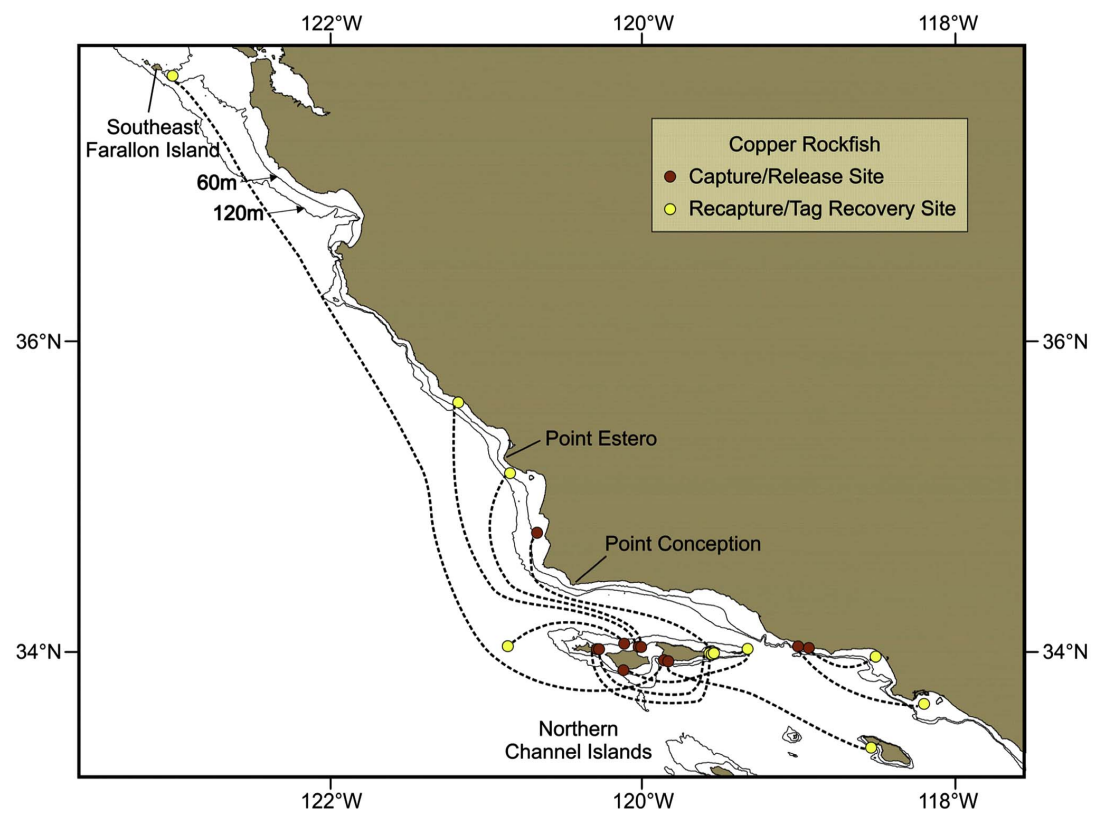

Fig. (6). Tag and recovery sites of eleven copper rockfish (Sebastes caurinus; 7 at size of maturity, 4 immature) with movements at least $50 \mathrm{~km}$.

Fourteen $(37 \%)$ of the recaptured blue rockfish (S. mystinus) were within $1 \mathrm{~km}$ of their tag/release site ( $74 \%$ within $5 \mathrm{~km}$ of tag/release sites; 42 - 688 DAL). Long distance movement was observed for an immature $[4,33]$ blue rockfish tagged between Santa Cruz and Anacapa Islands and recaptured in nearshore waters off Santa Monica $(92 \mathrm{~km}, 544$ DAL, Fig. 5, Table 4).

Seven $(39 \%)$ of the recaptured starry rockfish (S. constellatus) were within $1 \mathrm{~km}$ of the tag/release site (67\% within 5 $\mathrm{km}$ their tag/release sites; $30-1,263 \mathrm{DAL}$ ). Long distance movement was observed for a mature [33] starry rockfish tagged off San Clemente Island, recaptured nearshore off Redondo Beach (102 km, 740 DAL, Fig. 5, Table 4).
Long distance movements were observed for copper rockfish recaptured between coastal and offshore island areas, and among offshore islands (Fig. 6). Of 81 copper rockfish recaptured, $62(76 \%)$ were recaptured within $5 \mathrm{~km}$ of the tag/release sites (49\% within $1 \mathrm{~km}$ of the tag/release site; 11 - 1,513 DAL). Two mature $[4,33,36]$ copper rockfish, tagged off Santa Cruz Island were recaptured northward off the central California coast at $164 \mathrm{~km}(67 \mathrm{DAL})$ and $220 \mathrm{~km}$ (1,053 DAL). Mature copper rockfish tagged between Santa Rosa and San Miguel Islands and off the central California coast were recaptured in the same State Marine Reserve off Santa Cruz Island. Of copper rockfish tagged off Gull Island on the west side of Santa Cruz Island, an immature [4, 33, 36] fish was recaptured southward $153 \mathrm{~km}(1,085 \mathrm{DAL})$ at 


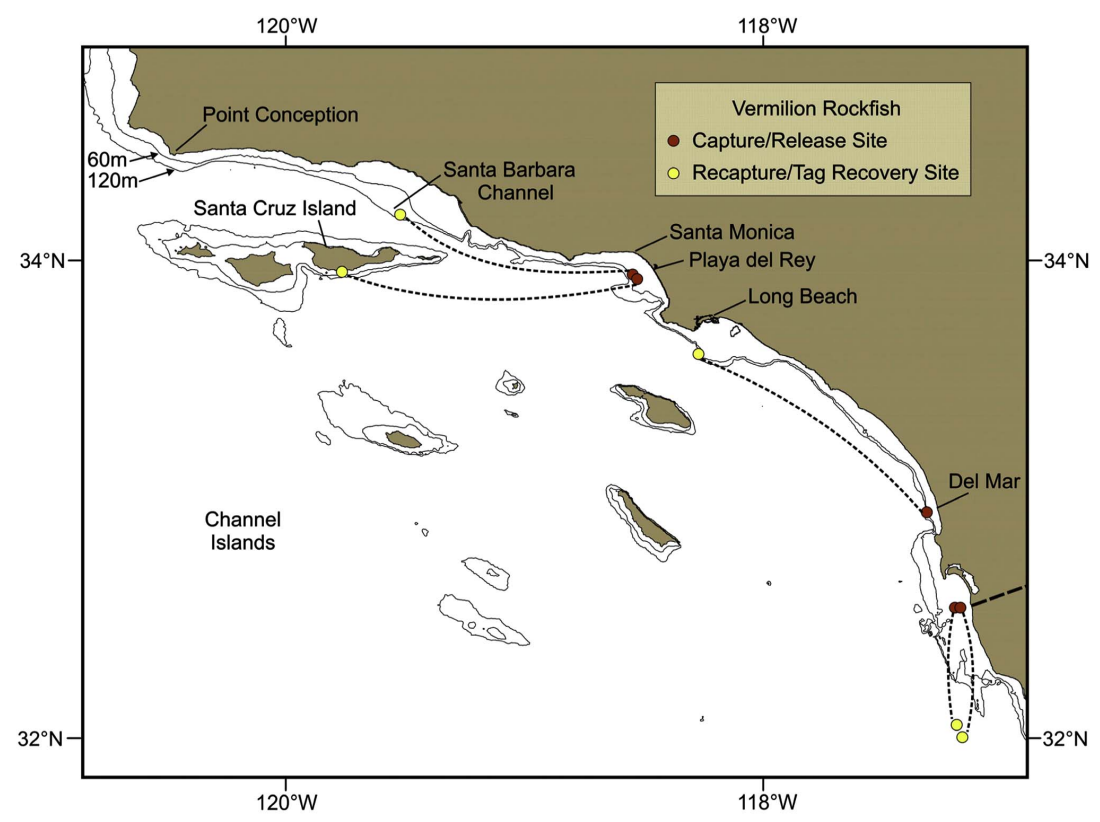

Fig. (7). Tag and recovery sites of 5 mature vermilion rockfish (Sebastes miniatus) that moved at least 50.



Fig. (8). Tag and recovery sites of 7 olive rockfish (Sebastes serranoides; 2 mature, 5 immature).

Santa Catalina Island and another immature was recaptured northward $488 \mathrm{~km}(1,222 \mathrm{DAL})$ at Southeast Farallon Island off San Francisco (Fig. 6, Table 4).

Of 51 recaptured vermilion rockfish, 38\% were recaptured less than $1 \mathrm{~km}$ from their original tag/release sites (72\% within $5 \mathrm{~km} ; 3$ - 1,694 DAL). Five mature [4, 32] vermilion were recaptured at long distances along coastal areas and at the offshore islands (Fig. 7). Two immature vermilion rockfish were tagged off Playa del Rey; one was recaptured off Santa Cruz Island (114 km, 419 DAL) and the other in the Santa Barbara Channel (96 km, 384 DAL, Fig. (7), Table 4).

Of 101 lingcod recaptured, $77 \%$ were within $5 \mathrm{~km}$ of the tag/release site (1 - 160 DAL); $51 \%$ of all lingcod recaptured were less than $1 \mathrm{~km}$ from tag/release site. An immature [37] lingcod was tagged in nearshore waters north of Oceanside and recaptured $153 \mathrm{~km}$ (475 DAL) northward off Malibu (Table 4).

Of 98 recaptured olive rockfish, $68 \%$ were within $1 \mathrm{~km}$ of the original tag/release site (2 - 396 DAL); however, olive rockfish were also recaptured at long distances $(50-510 \mathrm{~km}$, Fig. 8). Movement was confirmed from the nearshore coastal waters to offshore islands, from offshore islands to nearshore areas, and among offshore islands. A mature [4, 38] olive, tagged north of San Miguel Island, was recaptured off Carlsbad (308 km, 625 DAL); an immature olive was tagged off Santa Cruz Island and recaptured off Point Pinos near Monterey, California (359 km, 653 DAL). A mature [4, 38] olive, tagged off Santa Cruz Island, was recaptured at Middle Farallon Island off San Francisco (510 km, 342 DAL). 
This was longest recapture distance of any fish we tagged (Fig. 8, Table 4).

\section{DISCUSSION}

\section{Observed Mortality Rate and Effects of Handling}

The low observed mortality rate in this study was consistent with those of Shaw et al. [30]. They used different tagging methodologies on rockfish off Vancouver Island, Canada and, similarly to this study, found higher pre-tagging mortality $(5 \%)$ than post-tagging $(1.9 \%)$. They had higher mortality rates of $21 \%$ pre-tagging and $12.9 \%$ post-tagging at Queen Charlotte Sound which were attributed to smaller sample sizes, but could have been associated with physiological or behavioral differences in species tagged.

Researchers have found species-specific responses to barotrauma [39-41]. Hannah and Matteson [40] used in situ video to examine the behavioral effects of barotrauma resulting from hook-and-line captures at 12-124 $\mathrm{m}$ depth for 9 rockfish species and found behavioral impairment to be depth related but variable among species. They documented high re-submergence success rates for black, yellowtail, and quillback rockfish (S. maliger) across all depths sampled, and concluded that catch and release of these species did not result in high rates of short-term mortality. Because of high catch and release mortality rates in blue rockfish, yelloweye, and canary rockfish, they did not expect these fish likely to survive discard (catch and release). Jarvis and Lowe [41] found short-term survival varied by species from $36-82 \%$ and that condition at capture was not the best predictor of short-term survival in rockfish. They concluded that shortterm survival of rockfish increased with decreased surface holding time.

Post-release responses to capture and handling, including effects on recovery rates and potential mortality are unknown in this study, but we note that handling time was kept to a minimum. We acknowledge that displacement resulting from capture and release may have affected movement of some of our tagged fish.

\section{Site Fidelity}

We found limited motility over time for most rocky reef species in nearshore and offshore island areas $(5 \mathrm{~km}$ median recapture distance). We infer that most groundfish movement/motility or home range is confined to nearby rocky reef habitat $[4,25,37,42,43]$ which is consistent with other groundfish studies [44-46]. However, we also observed long range movements not previously detected which indicates the need for this type of large scale research project to better understand groundfish behavior, movements, and habitat needs.

Various natural rocky reef $[11,12]$ and artificial areas (e.g., pipeline, oil platforms) [47-51] provide important habitat and opportunity for residency to rockfish assemblages in temperate waters off California. Recently, Lowe et al., [21] examined movement patterns and site fidelity for 15 groundfish species including both shallow and deep shelf rockfish, cabezon, and lingcod in relation to 3 Santa Barbara Channel oil platforms. Acoustic telemetry was used to monitor 100 fish over a study period of 655 - 714 DAL. After 6 days,
$30 \%$ of tagged fish were not detectable, which was attributed primarily to post release mortality and emigration. The majority of fish tagged were vermilion $(n=61)$ and, in this study, the most mobile. Although sample sizes of other species were small, and the authors noted the likelihood of individual variation in site fidelity, site fidelity was highest for flag rockfish (S. rubrivinctus) treefish, widow rockfish, and lingcod which exhibited the highest site fidelity in the study $71 \%$ [20]. Lowe et al., [21] found that site fidelity near oil platforms was moderate for cabezon, rosy rockfish, and brown rockfish, and was lowest for bocaccio and for blue, greenspotted, starry, vermilion, and copper rockfish.

\section{Long Distance Movements/Motility}

We report copper rockfish of mature size that moved as far as $220 \mathrm{~km}$, in contrast to previous tagging studies that have indicated very limited movement $(2.8 \mathrm{~km}$ or less) [36, 37]. We also report new maximum movement/motility distances from two mature olive rockfish (308 and $510 \mathrm{~km}$ ). Love et al. [4] and Love [24] reported a maximum recovery distance of 20 miles $(33 \mathrm{~km})$ for olive rockfish. Long distance $(>50 \mathrm{~km})$ movement/motility is likely to have implications for definition of stock discreetness. While percentages of tagged fish recaptured at long distances in this study were relatively small, this movement/motility provides important insight into fish biology and habitat use which is important when considering Marine Protected Area size and spacing.

We observed inshore and offshore long distance movement/motility of mature scorpionfish, which may be seasonally related to reproduction. Love et al., [31] and Hartmann [36] suggested that California scorpionfish migrate to traditional grounds for spawning and remain there for brief periods.

As observed in this study, traditional tagging has shown that lingcod remain very close to tag/release sites [20, 37, 43, 52], but are known to make extensive migratory movements related to spawning [41, 53]. Lea et al., [43] reported one recaptured lingcod at $124 \mathrm{~km}$ from the tag/release site. This species has been tracked, using acoustic tagging, moving up to 239 miles $(385 \mathrm{~km})$ [53].

Long distance movement/motility (beyond home range) has been reported for shallow demersal and shallow shelf rockfish, as well as, deeper water semi-pelagic rockfish [5456]. This movement/motility has generally been attributed to ontogenetic shift: brown $[4,42,56]$, vermilion [20], yellowtail rockfish [54], and bocaccio [36, 51]. We assume some movement/motility that we detected could be attributed to ontogenetic shift as we focused on catching fish for tagging in shallow waters (mean depth $=29 \mathrm{~m}$; range 4 to $116 \mathrm{~m}$ ).

We observed long distance movement/motility of mature fish of six species of rockfish (brown, copper, gopher, olive, starry, and vermilion). While $50 \%$ of the brown rockfish tagged in the present study were recaptured within $1 \mathrm{~km}$ of their tag/release sites, six mature fish were recaptured at distances up to $90 \mathrm{~km}$ (see Table 4).

Long distance movement/motility of two mature gopher rockfish was observed with one recaptured among the Channel Islands $(50 \mathrm{~km})$ and one tagged/released offshore at Santa Rosa Island and recaptured off the central California 
coast $(110 \mathrm{~km}$, see Table 5). Previously, only movements of less than $3 \mathrm{~km}$ have been documented for gopher rockfish $[43,57]$.

Movement/motility of starry rockfish was previously little known. We documented a mature starry rockfish recapture at a minimum of $100 \mathrm{~km}$ from offshore San Clemente Island to nearshore off the southern California coast.

\section{CONCLUSIONS}

Using tag returns, this study confirms that large sample sizes are needed to reveal the wide range of fish movement patterns. Our initial captures and tag-recaptures indicate specific regions are preferred habitat for rockfish species. Such areas are: between San Miguel and Santa Rosa Islands, Santa Cruz and Anacapa Islands, and State Marine Reserves on the east and west sides of Santa Rosa and Santa Cruz Islands. This may also indicate that fish require use of more than one such prime habitat during a lifetime. Fish moved, some over long distances, to shared recapture sites, as seen in copper and olive rockfish. Movement between offshore and inshore habitat such as movement from the Channel Islands to nearshore central and southern California are indicative of specific importance of particular areas.

We observed that groundfish move among areas of varying ecological importance and that the use of multiple areas by individual fishes underscores the importance of fisheries and spatial management to protect and improve yield and to ensure conservation of rockfish and other groundfish species. Finally, we infer from tagging results that long distance movements may be more prevalent and probably important to groundfish species than previously assumed.

\section{CONFLICT OF INTEREST}

None declared.

\section{ACKNOWLEDGEMENTS}

We thank the WCFHS vessel captains (especially J. Diamond, F/V Stardust; J. Cacciola, F/V Sea Star; D. Wortham, F/V Erna B; S. Peterson, F/V Point Loma; B. Valney, F/V Seabiscuit, J. Helgren, F/V Advance, P. Cavanaugh, F/V Pacific Dawn, and J. Villareal, F/V Mirage) and the many volunteer anglers who facilitated capture of the large number of fish tagged in this study. Additional thanks are extended to R. C. Fletcher and L. B. Boydstun for their help and encouragement. We thank California Department of Fish and Game, CDFG, in particular E. Roberts, S. Owen, and M. Connell, for assistance with developing the study and with collection of tag return data. T. Buck, J. Duran, K. Lampson, E. Miller, T. Masson were field tagging technicians and we thank them for their excellent work at sea. R. Hilborn, R. Parrish, and C. J. Walters provided insightful suggestions. M. Hinton greatly improved this document with perceptive editorial recommendations. D. Losey, NMFS, SWFSC, kindly provided research documents. R. Allen, NMFS, SWFSC, provided graphics work and mapping data.

This study was primarily funded by the Congressional Groundfish Disaster Relief Fund and The West Coast Cooperative Research Program, administered by Pacific States
Marine Fisheries Commission as contracts and grants. Each tagging technician and scientist outside CDFG obtained their own California Scientific Collector's Permit for the at-sea tagging research.

\section{REFERENCES}

[1] Hubbs CL, Follett WI, Dempster LJ. List of the fishes of California. Calif Acad Sci Occas Pap 1979; 133: 1-51.

[2] Hyde JR, Vetter RD. The origin, evolution, and diversification of rockfishes of the genus Sebastes (Cuvier). Mol Phylogenet Evol 2007; 44:790-811.

[3] Yoklavich M, Greene HG, Cailliet GM, Sullivan DE, Lea RN, Love MS. Habitat associations of deep-water rockfishes in a submarine canyon: an example of a natural refuge. Fish Bull 2000; 98: 625-41.

[4] Love MS, Yoklavich M, Thorsteinson L. The rockfishes of the northeast Pacific. Berkeley, California: University of California Press 2002.

[5] Love MS, Yoklavich M, Schroeder DM. Demersal fish assemblages in the southern California Bight based on visual surveys in deep water. Environ Biol Fish 2009; 84: 55-68.

[6] Parker SJ, Berkeley SA, Golden JT, et al. Management of Pacific rockfish. Fisheries 2000; 25: 22-30.

[7] Lucas S. History and status of commercial live fish fisheries in California and the United States west coast. Live Reef Fish Inform Bull 2006; 16: 19-25.

[8] Punt AE, Ralston S. A management strategy evaluation of rebuilding revision rules for overfished rockfish stocks. In: Heifetz J, DiCosmo J, Gharrett AJ, Love MS, O'Connell VM, Stanley RD, Eds. Biology, assessment, and management of North Pacific rockfishes. AK Sea Grant College Prog AK-SG-07-01 2007; pp. 329-51.

[9] PFMC. Pacific Coast groundfish fishery management plan for the California, Oregon, and Washington groundfish fishery as amended through Amendment 19 including Amendment 15. Portland OR, Pacific Fishery Mgt Council 2008.

[10] CDFG [Homepage on Internet] 2011-2012 Ocean sport fishing regulations. Sacramento; California Department of Fish and Game 2011. Available from: http://www.dfg.ca.gov/marine/groundfishcentral/

[11] Hilborn R. Ecosystem-based fisheries management: the carrot or the stick? Mar Ecol-Prog Ser 2004; 274: 275-8.

[12] Gunderson DR, Vetter RD. In: Kritzer JP, Sale PF, Eds. Marine metapopulations. Amsterdam: Academic Press 2006; pp. 69-117.

[13] Gunderson DR, Parma AM, Hilborn R, et al. The challenge of managing nearshore rocky reef resources. Fisheries 2008; 33: 1729.

[14] Copps SL, Yoklavich MM, Parkes G, et al. Applying marine habitat data to fishery management on the US west coast: Initiating a policy-science feedback loop. In: Todd BJ, Greene HG, Eds. Mapping the seafloor for habitat characterization: Canada: Geological Association of Canada 2007; pp. 47: 439-450

[15] Starr RM, Heine JN, Johnson KA. Techniques for tagging and tracking deep-water rockfishes. N Am J Fish Manage 2000; 20: 597-609.

[16] Lowe CG, Bray RN. In: Allen LG, Pondella II, Horn MH, Eds. The ecology of California marine fishes: California and adjacent waters. California: University of California Press 2006; pp. 524-553

[17] Lowe CG. Movement and activity patterns of rockfish In: Berntson EA, Levin PS, Moran PC, Eds. Conservation of North Pacific rockfishes: ecological genetics and stock structure. Proceedings of the workshop Seattle: Washington March 2004. NOAA Technical Memorandum. NMFS-NWFSC-80 2004; pp. 17-20.

[18] Parrish FA, Moffitt RB. Subsurface fish handling to limit decompression effects on deepwater species. Mar Fish Rev 1993; 54: 2932.

[19] Sigurdsson T, Thorsteinsson V, Gústafsson L. In situ tagging of deep-sea redfish: application of an underwater tagging system. ICES J Mar Sci 2006; 63: 523-31.

[20] Topping DT, Lowe CG, Caselle JE. Home range and habitat utilization of adult California sheephead, Semicossyphus pulcher (Labridae), in a temperate no-take marine reserve. Mar Biol 2005; 147: 301-11.

[21] Lowe CG, Anthony KM, Jarvis ET, Bellquist LF, Love MS. Site fidelity and movement patterns of groundfish associated with off- 
shore petroleum platforms in the Santa Barbara Channel. Mar Coast Fish: Dyn Mgt Ecosyst Sci 2009; 1: 71-89.

[22] Tolimieri N, Andrews K, Williams G, Katx S, Levin PS. Home range size and patterns of space use by lingcod, copper rockfish and quillback rockfish in relation to diel and tidal cycles. Mar Ecol Prog-Ser 2009; 380: 229-43.

[23] Gotshall DW. Increasing tagged rockfish (genus Sebastodes) survival by deflating the swim bladder. CDFG Fish Bull 1964; 50: 253-60.

[24] Love MS. Isolation of olive rockfish, Sebastes serranoides, populations off southern California. Fish Bull 1980; 77: 975-83.

[25] Kahn RG, Pearson DE, Dick EJ. Comparison of standard length, fork length, and total length for measuring west coast marine fisheries. Mar Fish Rev 2004; 66: 31-3.

[26] Gurtin S, Brown ML, Scalet CG. Retention of Floy FD-94 anchor tags and effect on growth and condition of northern pike and largemouth bass. J Freshwater Ecol 1999; 14: 281-6.

[27] Scholten GD, Isermann DA, Willis DW. Retention and survival rates associated with the use of t-bar anchor tags in marking yellow perch. Proc S D Acad Sci 2002; 81: 35-8.

[28] Livings ME, Schoenebeck CW, Brown ML. Long-term anchor tag retention in yellow perch, Perca flavescens (Mitchill). Fish Manage Ecol 2007; 14: 365-6.

[29] Shaw W, Nagtegall DA, Archibald CP, Leaman BM. Rockfish tagging cruises off southwest Vancouver Island (M/V Ocean King) and off Northwest Vancouver Island and in Queen Charlotte Sound (M/V Blue Waters) during 1980. Canadian Data Report of Fisheries and Aquatic Sciences No. 2881981.

[30] Ugoretz J. Final 2002 environmental document: marine protected areas in the National Oceanic and Atmospheric Administration's Channel Islands National Marine Sanctuary, Volume 1. Sacramento: California Department of Fish and Game 2002. Available from: http://www.dfg.ca.gov/mrd/channel_islands

[31] Love MS, Axell B, Morris P, Collins R, Brooks A. Life history and fishery of the California scorpionfish, Scorpaena guttata, within the southern California Bight. Fish Bull 1987; 85: 99-116.

[32] Love MS, Morris P, McCrae M, Collins R. Life history aspects of 19 rockfish species (Scorpaenidae: Sebastes) from the southern California Bight. US Commerce Association. NOAA Technical Report NMFS-87 1990.

[33] Wyllie Echeverria T. Thirty-four species of California rockfishes: maturity and seasonality of reproduction. Fish Bull 1987; 85: 22950.

[34] Love MS, Johnson K. Aspects of the life histories of grass rockfish Sebastes rastrelliger, and brown rockfish, S. auriculatus, from southern California. Fish Bull 1998; 87: 100-9.

[35] Rothrock GC. Age-length, weight, fecundity, and meristics of the kelp greenling (Hexagrammos decagrammus) off California. M.S. Thesis. Davis CA: University of California Davis 1982.

[36] Love MS. Probably more than you wanted to know about the fishes of the Pacific coast. Santa Barbara: Really Big Press 2006.

[37] Miller DJ, Geibel JJ. Summary of blue rockfish and lingcod life histories; a reef ecology study; and giant kelp, Macrocystis pyrifera, experiments in Monterey Bay, California. CDFG Fish Bull 1973; 158: 1-135.

[38] Love MS, Westphal W. Growth, reproduction, and food habits of olive rockfish, Sebastes serranoides, off central California. Fish Bull 1981; 79: 533-45.
[39] Pribyl AL, Schreck CB, Kent ML, Parker SJ. The differential response to decompression in three species of nearshore Pacific rockfish. N Am J Fish Manage 2009; 29: 1479-86.

[40] Hannah RW, Matteson KM. Behavior of nine species of Pacific rockfish after hook-and-line capture, recompression, and release. $\mathrm{T}$ Am Fish Soc 2007; 136: 24-33.

[41] Jarvis ET, Lowe CG. The effects of barotrauma on the catch-andrelease survival of southern California nearshore and shelf rockfish (Scorpaenidae, Sebastes spp.). Can J Fish Aquat Sci 2008; 65: 1286-96.

[42] Hartmann AR. Movement of scorpionfishes (Scorpaenidae: Sebastes and Scorpaena) in the southern California Bight. CDFG Fish Bull 1987; 73: 68-79.

[43] Lea RN, McAllister RD, VanTresca DA. Biological aspects of nearshore rockfishes of the genus Sebastes from central California with notes on ecologically related sport fishes. CDFG Fish Bull 1999; 177: 1-112.

[44] Matthews KR. An experimental study of the habitat preferences and movement patterns of copper, quillback and brown rockfish (Sebastes spp.). Environ Biol of Fish 1990a; 29: 161-78.

[45] Matthews KR. A telemetric study of home ranges and homing routes of copper and quillback rockfishes on shallow rocky reefs. Can J Zool 1990b; 68: 2243-50.

[46] Matthews KR. A comparative study of habitat use of young-of-theyear, subadult, and adult rockfishes on four habitat types in central Puget Sound. Fish Bull 1990c; 88: 223-39.

[47] Carlisle JG Jr., Turner CH, Ebert EE. Artificial habitat in the marine environment. CDFG Fish Bull 1964; 124:1-93.

[48] Caselle JE, Love MS, Fusaro C, Schroeder D. Trash or habitat? Fish assemblages on offshore oilfield seafloor debris in the Santa Barbara Channel, California. ICES J Mar Sci 2002; 59: S258-65.

[49] Helvey M. Are southern California oil and gas platforms essential fish habitat? ICES J Mar Sci 2002; 59: S266-71.

[50] Schroeder DM, Love, MS. Ecological and political issues surrounding decommissioning of offshore oil facilities in the southern California Bight. Ocean Coast Manage 2004; 47: 21-48.

[51] Love MS, Schroeder DM, Lenarz W, MacCall A, Bull AS, Thorsteinson L. Potential use of offshore marine structures in rebuilding an overfished rockfish species, bocaccio (Sebastes paucispinis). Fish Bull 2006; 104: 383-90.

[52] Mathews SB, LaRiviere M. Movement of tagged lingcod, Ophiodon elongatus, in the Pacific Northwest. Fish Bull 1987; 85: 153-9.

[53] Matthews KR. A telemetry study of the home ranges and homing routes of lingcod Ophlodon elongatus on shallow rocky reefs off Vancouver Island, British Columbia. Fish Bull 1992; 90: 784-90.

[54] Mathew SB, Barker MW. Movements of rockfish (Sebastes) tagged in northern Puget Sound, Washington. Fish Bull 1983; 82: 916-22.

[55] Culver BN. Results of tagging black rockfish (Sebastes melanops) off the Washington and northern Oregon coast. In: Melteff BR, Ed. Proceedings of the International Rockfish Symposium. University of Alaska Sea Grant AK-SG-87-02 1987; pp. 231-285.

[56] Stanley RD, Leaman BM, Haldorson L, O'Connell VM. Movements of tagged adult yellowtail rockfish, Sebastes flavidus, off the west coast of North America. Fish Bull 1994; 92: 655-63.

[57] Matthews KR. Movement of two nearshore, territorial rockfishes previously reported as non-movers and implications to management. CDFG Bull 1986; 72: 103-9.

(C) Hanan and Curry; Licensee Bentham Open.

This is an open access article licensed under the terms of the Creative Commons Attribution Non-Commercial License (http://creativecommons.org/licenses/ by-nc/3.0/) which permits unrestricted, non-commercial use, distribution and reproduction in any medium, provided the work is properly cited. 